\title{
Pricing European Call Currency Option Based on Fuzzy Estimators
}

\author{
Xing Yu, Hongguo Sun, Guohua Chen \\ The Department of Mathematics \& Applied Mathematics, Humanities \& Science and Technology \\ Institute of Hunan Loudi, Loudi, China \\ E-mail: hnldyu@sina.com, hnyuxing@163.com \\ Received December 30, 2010; revised March 2, 2011; accepted March 5, 2011
}

\begin{abstract}
In this paper we present an application of fuzzy estimators method to price European call currency option. We make use of fuzzy estimators for the volatility of exchange rate which based on statistical data to obtain the fuzzy pattern of G-K model. A numerical example is presented to get the $\alpha$-level closed intervals of the European call currency option fuzzy price.
\end{abstract}

Keywords: Currency Option, Fuzzy Estimators, Fuzzy Volatility, G-K Model

\section{Introduction}

In financial markets there are great fluctuations, thus the element of vagueness and uncertainty is frequent. For instant, on the foreign exchange market, the spot exchange rates fluctuate from time to time according to the financial market effects and may occur imprecisely; therefore, it is natural to consider the fuzzy foreign interest rate and the domestic interest rate, fuzzy volatility and fuzzy exchange rate. Practically, many financial investors are concerned with the currency options price range, then at the last of the paper, we get the $\alpha$-level close intervals of the European call currency option fuzzy price.

Since the closed form solution of the European currency options piecing model was derived by Garman and Kohlhagen (1983) based on Black and Scholes (1973), many methodologies for the currency options pricing have been proposed by using modification of GarmanKohlhagen (G-K) model, such as Amin and Jarrow (1991), Heston (1993), Bates (1996), Ekvall et al. (1997), Lim et al. (1998), Rosenberg (1998), Sarwar and Krenhbiel (2000) [1], Bollen and Rasiel (2003) [2]. In this paper we present an application of fuzzy estimators method to price European call currency option. We make use of fuzzy estimators for the volatility of exchange rate which based on statistical data to obtain the fuzzy pattern of G-K model. We use the fuzzy estimators based on confindence intervals introduced by Papadopoulos and Sfiris in [3] in order to estimate the volatility of exchange rate having sample data. This paper is organized as follows.
In Section 2, the notions of fuzzy number and the arithmetics of fuzzy numbers are introduced. In Section 3, the fuzzy patterns of G-K model. In Section 4, the optimal model is provided in order to obtain the belief degrees of given option price. Finally, the empirical study is performed.

\section{Fuzzy Sets Preliminaries}

\subsection{Fuzzy Set Theory}

Now we remind some facts about fuzzy sets and numbers [4]:

Let $\mathrm{X}$ be a universal set and $\tilde{A}$ be a fuzzy subset of $\mathrm{X}$, we denote by $\mu_{\overline{\mathrm{A}}}$ its membership function

$\mu_{\bar{A}}: X \rightarrow[0,1]$, and by $\tilde{A}_{\alpha}=\left\{x: \mu_{\bar{A}} \geq \alpha\right\}$ the $\alpha$-level set of, where is the closure of the set. Let $\tilde{a}$ be a fuzzy number. Then, under our assumptions, the $\alpha$-level set $\tilde{a}_{\alpha}$ is a closed interval, which can be denoted by $\tilde{a}_{\alpha}=\left[\tilde{a}_{\alpha}^{L}, \tilde{a}_{\alpha}^{U}\right]$.

The four arithmetic operations on closed intervals are defined as follows:

$$
\begin{gathered}
{[a, b]+[d, e]=[a+d, b+e]} \\
{[a, b]-[d, e]=[a-e, b-d]} \\
{[a, b] \cdot[d, e]=[\min \{a d, a e, b d, b e\}, \min \{a d, a e, b d, b e\}]} \\
\text { and provided that } 0 \notin[d, e]
\end{gathered}
$$


if $a, b, d, e>0$, then $[a, b] \cdot[d, e]=[a d, b e]$

$[a, b] /[d, e]=[a, b] \cdot\left[\frac{1}{d}-\frac{1}{e}\right]$ and provided that $0 \notin[d, e]$

\subsection{Triangular Fuzzy Number}

The membership function of a triangular fuzzy number $\tilde{a}$ is defined by:

$$
\mu_{\bar{a}}(x)=\left\{\begin{array}{lc}
\left(x-a_{L}\right) /\left(a_{c}-a_{L}\right), & \text { if } a_{L} \leq x \leq a_{C} \\
\left(a_{R}-x\right) /\left(a_{R}-a_{C}\right), & \text { if } a_{C}<x \leq a_{R} \\
0, & \text { otherwise }
\end{array}\right.
$$

Which is denoted by $\tilde{a}=\left(a_{L} ; a_{C} ; a_{R}\right)$.The $\alpha$-level set of $\tilde{a}$ is then:

$$
\begin{gathered}
\tilde{a}_{\alpha}=\left[(1-\alpha) a_{L}+\alpha a_{C},(1-\alpha) a_{R}+\alpha a_{C}\right] \\
\tilde{a}_{\alpha}^{L}=(1-\alpha) a_{L}+\alpha a_{C}, \quad \tilde{a}_{\alpha}^{U}=(1-\alpha) a_{R}+\alpha a_{C}
\end{gathered}
$$

\section{Fuzzy Currency Options Pricing Model}

\subsection{Estimate Fuzzy Volatility from Fuzzy Estimators of $\sigma^{2}$-Fuzzy Estimators Based on Confidence Intervals}

Following the proposition proved by Papadopoulos and Sfiris in [3], we consider the fuzzy volatility as:

$$
\sigma^{2}(x)=\left\{\begin{array}{l}
\frac{2-\beta}{1-\beta}-\frac{2}{1-\beta} \Phi\left(\sqrt{\frac{n-1}{2}}\left(\frac{s^{2}}{x}-1\right)\right) \text { if } \frac{s^{2}}{1+\Phi^{-1}\left(1-\frac{\beta}{2}\right) \sqrt{\frac{2}{n-1}}} \leq x \leq s^{2} \\
\frac{2-\beta}{1-\beta}-\frac{2}{1-\beta} \Phi\left(\sqrt{\frac{n-1}{2}}\left(1-\frac{s^{2}}{x}\right)\right) \text { if } s^{2} \leq x \leq \frac{s^{2}}{1-\Phi^{-1}\left(1-\frac{\beta}{2}\right) \sqrt{\frac{2}{n-1}}}
\end{array}\right.
$$

its $\alpha$-cut is the following:

$$
\sigma^{2}[\alpha]=\left[\frac{s^{2}}{1+u \sqrt{\frac{2}{n-1}}}, \frac{s^{2}}{1-u \sqrt{\frac{2}{n-1}}}\right]
$$

where

$$
u=\Phi^{-1}\left(1-\left(\frac{1}{2}-\frac{\beta}{2}\right) \alpha-\frac{\beta}{2}\right)
$$

\subsection{The G-K Model and Its Fuzzy Pattern}

The G-K model for a European call currency option with expiry date $T$ and strike price $K . s_{t}$ denotes the spot exchange rate at time $t \in[0, T]$, and $c_{t}$ denotes the price of a currency option at time $t$. The G-K model is as:

$$
\begin{aligned}
& c_{t}=s_{t} e^{-r_{1} \tau} N\left(d_{1}\right)-K e^{-r_{2} \tau} N\left(d_{2}\right), \tau=T-t \\
& d_{1}=\left[\ln \left(s_{t} / K\right)+\left(r_{2}-r_{1}+\sigma^{2} / 2\right) \tau\right] /(\sigma \sqrt{\tau}), \\
& d_{2}=d_{1}-\sigma \sqrt{\tau}
\end{aligned}
$$

where $r_{1}$ denotes the foreign interest rate, $r_{2}$ denotes the domestic interest rate, $\sigma$ denotes the volatility, and $\mathrm{N}$ stands for the cumulative distribution function of a standard normal random variable $N(0,1)$.

Under the considerations of fuzzy exchange rate $\tilde{s}_{t}$, fuzzy interest rate $\tilde{r}_{1}, \tilde{r}_{2}$, fuzzy volatility $\tilde{\sigma}$, the fuzzy price is denoted as $\tilde{c}_{t}$. The fuzzy patterns of G-K model are described as the interval:

$$
\begin{aligned}
& \left(\tilde{c}_{t}\right)_{\alpha}^{L}=\left(\tilde{s}_{t}\right)_{\alpha}^{L} e^{-\left(\tilde{r}_{1}\right)_{\alpha}^{U} \tau} N\left(\left(\tilde{d}_{1}\right)_{\alpha}^{L}\right)-K e^{-\left(\tilde{r}_{2}\right)_{\alpha}^{L} \tau} N\left(\left(\tilde{d}_{2}\right)_{\alpha}^{U}\right) \\
& \left(\tilde{c}_{t}\right)_{\alpha}^{U}=\left(\tilde{s}_{t}\right)_{\alpha}^{U} e^{-\left(\tilde{r}_{1}\right)_{\alpha}^{L} \tau} N\left(\left(\tilde{d}_{1}\right)_{\alpha}^{U}\right)-K e^{-\left(\tilde{r}_{2}\right)_{\alpha}^{U} \tau} N\left(\left(\tilde{d}_{2}\right)_{\alpha}^{L}\right)
\end{aligned}
$$

where

$$
\begin{aligned}
& \left(\tilde{d}_{1}\right)_{\alpha}^{L}=\left[\ln \left(\left(\tilde{s}_{t}\right)_{\alpha}^{L} / K\right)+\left(\left(\tilde{r}_{2}\right)_{\alpha}^{L}-\left(\tilde{r}_{1}\right)_{\alpha}^{U}+\left((\tilde{\sigma})_{\alpha}^{L}\right)^{2} / 2\right) \tau\right] /\left((\tilde{\sigma})_{\alpha}^{U} \sqrt{\tau}\right) \\
& \left(\tilde{d}_{1}\right)_{\alpha}^{U}=\left[\ln \left(\left(\tilde{s}_{t}\right)_{\alpha}^{U} / K\right)+\left(\left(\tilde{r}_{2}\right)_{\alpha}^{U}-\left(\tilde{r}_{1}\right)_{\alpha}^{L}+\left((\tilde{\sigma})_{\alpha}^{U}\right)^{2} / 2\right) \tau\right] /\left((\tilde{\sigma})_{\alpha}^{L} \sqrt{\tau}\right)
\end{aligned}
$$


Table 1. The price intervals for a given belief degree.

\begin{tabular}{cccccc}
\hline$\alpha$ & $\tilde{c}_{\alpha}$ & $\alpha$ & $\tilde{c}_{\alpha}$ & $\alpha$ & $\tilde{c}_{\alpha}$ \\
\hline 0.8 & {$[0.5475,0.5502]$} & 0.87 & {$[0.5479,0.5497]$} & 0.94 & {$[0.5484,0.5492]$} \\
0.81 & {$[0.5475,0.5501]$} & 0.88 & {$[0.5480,0.5496]$} & 0.95 & {$[0.5484,0.5491]$} \\
0.82 & {$[0.5476,0.5500]$} & 0.89 & {$[0.5480,0.5495]$} & 0.96 & {$[0.5486,0.5491]$} \\
0.83 & {$[0.5476,0.5499]$} & 0.9 & {$[0.5482,0.5495]$} & 0.97 & {$[0.5486,0.5490]$} \\
0.84 & {$[0.5477,0.5499]$} & 0.91 & {$[0.5482,0.5494]$} & 0.98 & {$[0.5487,0.5490]$} \\
0.85 & {$[0.5478,0.5498]$} & 0.92 & {$[0.5483,0.5494]$} & 0.99 & {$[0.5487,0.5488]$} \\
0.86 & {$[0.5479,0.5498]$} & 0.93 & {$[0.5483,0.5493]$} & 1.00 & {$[0.5488,0.5488]$} \\
\hline
\end{tabular}

$$
\begin{aligned}
& \left(\tilde{d}_{2}\right)_{\alpha}^{L}=\left(\tilde{d}_{1}\right)_{\alpha}^{L}-(\tilde{\sigma})_{\alpha}^{U} \sqrt{\tau} \\
& \left(\tilde{d}_{2}\right)_{\alpha}^{U}=\left(\tilde{d}_{1}\right)_{\alpha}^{U}-(\tilde{\sigma})_{\alpha}^{L} \sqrt{\tau}
\end{aligned}
$$

\subsection{To Calculation the Fuzzy Currency Option Steps}

Calculating the fuzzy currency option as the following steps:

Step 1. We compute the Estimate fuzzy volatility from fuzzy estimators of $\sigma^{2}$ based the history data following (1)-(2).

Step 2. On the hypothesis of the fuzzy interest and fuzzy exchange rate are triangular fuzzy numbers, and we obtain the $\alpha$-cuts of fuzzy currency option which are closed intervals following (1)-(2), (5)-(10).

\section{Empirical Example}

In this section, our model is tested with the daily market price data of EUR/CNY. These market data come from [5] and cover the period 9-27-2010 to 11-15-2010. We calculate $s^{2}=0.013287$, the spot exchange rate is around 9.2418, and assume the strike price is $\mathrm{K}=9.12$ with one year to expiry. Let $\beta=0.01$. The triangular fuzzy numbers are $\tilde{r}_{1}=(1.09 \%, 1.1 \%, 1.11 \%)$; $\tilde{r}_{2}=(2.4 \%, 2.5 \%, 2.6 \%) ; \tilde{s}_{t}=(9.2398,9.2418,9.2438)$.

Because the closed form of volatility interval based on samples are very hard to get, so we adopt numerical algorithm to solve optimization model. For example, we average the interval $[0.8,1]$ into 20 equal parts, that is $\alpha=0.8,0.81,0.82, \cdots, 1$. Based on every $\alpha$, we calculate the intervals of option price as Table 1, from which, we find that with $\alpha$ increasing ,the left interval increasing while the right interval decreasing, and both intersects when $\alpha=1$ where is the situation matches the actual price by Equations $(3,4)$. For $\alpha=0.95$, it means that the call currency option price will lie in the closed interval [0.5484, 0.5491] with belief degree 0.95 . From another point of view, if a financial analyst is comfortable with this belief degree 0.95, then he (she) can pick any value from the closed interval [0.5484, $0.5491]$ as the option price for his(her)later use.

\section{Conclusions}

Owing to the fluctuation of financial market from time to time, the data sometimes cannot be expected in a precise sense. Therefore, the fuzzy sets theory provides a useful tool for conquering this kind of impreciseness. The fuzzy pattern of G-K model is proposed in this paper. Under the considerations of fuzzy estimators for the volatility of exchange rate which based on statistical data, the European call currency option price turns into a fuzzy number. This makes the financial analyst who can pick any European call currency option with an acceptable belief degree for he (her) later use and get the interval for the price with the given belief degree.

\section{Acknowledgments}

Supported by Youth Fund of Hunan Institute of Humanities Science and technology (2008QN013).

\section{References}

[1] G. Sarwar and T. Krehbiel, "Empirical Performance of Alternative Piecing Models of Currency Options," The Journal of Futures Markets, Vol. 20, No. 2, March 2000, pp. 265-291. doi:10.1002/(SICI)1096-9934(200003)20:3<265::AID-F $\mathrm{UT} 4>3.0 . \mathrm{CO} ; 2-4$

[2] P. B. N. Bollen and E. Rasiel, "The Performance of Alternative Valuation Models in the OTC Currency Options Market," Journal of International Money and Finance, Vol. 22, No. 1, February 2003, pp. 33-64. 
doi:10.1016/S0261-5606(02)00073-6

[3] A. Thavaneswaran, J. Singh and S. S. Appadoo, "Option Pricing for Some Stochastic Volatility Models," The Journal of Risk Finance, Vol. 7, No. 4, 2006, pp. 425-445. doi:10.1108/15265940610688982
[4] G. J. Klir and B. Yuan, "Fuzzy Sets and Fuzzy Logic: Theory and Applications,” Prentice Hall, Englewood Cliffs, 1995.

[5] http://www.123cha.com/hl/?q=100\&from=EUR\&to=CN Y\&s=EURCNY\#symbol=EURCNY=X;range $=3 \mathrm{~m}$ 